\title{
Stable Heating Technology for Catalytic Combustion Hydrogen Gas Sensor Using Quartz Resonators
}

\author{
Hiroshi Oigawa, ${ }^{1 *}$ Mizuho Shimojima, ${ }^{1}$ Tooru Tsuno, ${ }^{2}$ and Toshitsugu Ueda ${ }^{2}$ \\ ${ }^{1}$ Kitakyushu Research Laboratory, KOA Corporation, \\ 2-1 Hibikino, Wakamatsu-ku, Kitakyushu-shi, Fukuoka 808-0135, Japan \\ ${ }^{2}$ IPS Research Center, Waseda University, 2-2 Hibikino, Wakamatsu-ku, Kitakyushu-shi, Fukuoka 808-0135, Japan
}

(Received January 10, 2018; accepted April 26, 2018)

Keywords: hydrogen, gas sensor, platinum catalyst, heater, temperature controller

In this paper, we focus on heating technologies for gas sensors. To discuss the requirements of heating accuracy of the sensor quantitatively, the effect of temperature error on the sensitivity of the sensor was evaluated. A Au thin film was used as a catalytically inactive heater for the sensor. The design and fabrication of this novel sensor with a $\mathrm{Au}$ thin-film heater is discussed. To stabilize the properties of the heater, the effect of annealing on the resistivity and temperature coefficient of resistance of the heater at $120{ }^{\circ} \mathrm{C}$ was verified by experiment. Additionally, a heater control circuit using op-amps was designed. Using the controller, accurate heating with a temperature error within $0.16{ }^{\circ} \mathrm{C}$ was obtained. As a result, constant sensitivity in a hydrogen gas concentration range of $0-2$ vol. $\%$ was provided, and the ability to detect a concentration of 0.01 vol. $\%$ was demonstrated.

\section{Introduction}

Along with technological developments for the realization of a hydrogen society, demands for hydrogen gas sensors are also increasing. Specifically, to use hydrogen gas safely as a fuel, leak detection is an indispensable technology. Because the explosion limit of hydrogen gas in air is 4 to 75 vol.\%, the sensitivity of leak detectors in the range of approximately 0.1 to 4 vol.\% is important. ${ }^{(1,2)}$ To detect 0.1 vol.\% hydrogen with high accuracy, a resolution of approximately 0.01 vol.\% is required. As hydrogen gas sensors, catalytic combustion types, semiconductive metal-oxide types, and thermal conductivity types have been studied and developed. Each type of sensor has a different concentration range for detection. Because they easily match the required detection concentration range, catalytic combustion sensors have been adopted for commercially available fuel cell vehicles. On the other hand, complementary combined sensor systems using catalytic combustion and semiconductive metal-oxides have been used in hydrogen filling stations, because commercially available catalytic combustion sensors have not achieved satisfactionary sensitivity at low concentration ranges.

*Corresponding author: e-mail: hi-oigawa@koanet.co.jp

http://dx.doi.org/10.18494/SAM.2018.1906 
A catalytic combustion hydrogen gas sensor detects heat generated by the reaction of hydrogen and oxygen molecules on the catalyst. In general, the catalytic activity of Pt-based catalysts can be enhanced by changing their temperature. Therefore, almost all gas sensors have a heater. As their temperature increases, the sensitivity of the gas sensors increases. Conventional sensors require heating from 250 to $550{ }^{\circ} \mathrm{C}^{(1-3)}$ The heating of sensors also causes the effects of environmental temperature and humidity changes to be reduced. ${ }^{(4)}$ Reducing the influence of water vapor generated in the catalytic reaction is also one of the objectives. For this reason, to achieve stable operation, the catalyst should be heated over $100{ }^{\circ} \mathrm{C}$. On the other hand, high-temperature operation introduces disadvantages such as large energy consumption and low gas selectivity. According to previous studies, gas selectivity depends on the temperature of the catalyst; thus, the improvement of selectivity is possible when the heating temperature is decreased. As an example, Shin et al. have reported hydrogen sensors with excellent gas selectivity heated at around $100{ }^{\circ} \mathrm{C} .{ }^{(5)}$

We are developing catalytic combustion hydrogen gas sensors using quartz resonators. ${ }^{(6-8)}$ The sensor uses resonance frequency temperature characteristics to detect the heat of reaction of hydrogen on the Pt catalyst. To improve the resolution of hydrogen concentration, a special cut quartz wafer, which achieved a large temperature coefficient of frequency (TCF), was used for the sensor. As a result, the sensor realized low-temperature operation of around $100{ }^{\circ} \mathrm{C} .{ }^{(6)}$ One of the resonators is the sensing resonator, the electrodes of which are coated with a Pt catalyst. The other is the reference resonator, the electrodes of which are made from catalytically inactive $\mathrm{Au}$, and it is used as a reference with respect to environmental deviations. The heater is patterned on the surface of both resonators. On the previously developed sensor, the heater was made from Pt thin film, because Pt is thermally and chemically stable for long periods. ${ }^{(8)}$ However, the Pt heater had caused fluctuations in the heating temperature during catalytic reactions. To improve the stability of the heating temperature, we proposed the application of a Au thin film to the heater of the sensor; the possibility of replacing Pt with a Au thin film has been reported. ${ }^{(9)}$ In this paper, we report the design and fabrication of a hydrogen sensor with a $\mathrm{Au}$ thin-film heater and the development of a heater controller to realize stable heating.

\section{Requirements of Gas Sensor Heating}

\subsection{Accuracy of temperature control}

In a previous work, heating methods for gas sensors have been developed. The simplest method is the Joule heating of a Pt catalyst by supplying current directly. ${ }^{(10)}$ Another example is a method that inserts a heater coil into Pt support ceramics, and this structure is widely used in commercially available sensors. ${ }^{(3)}$ For our sensor, a thin-film heater is located on the surface of the quartz resonators. Our sensor has a potential of gas while operating at a low temperature of around $100{ }^{\circ} \mathrm{C}$ because of its ability to detect temperature changes with high resolution. Conversely, the effect on the sensitivity due to variations in heating temperature is large. Therefore, the effect of heating temperature on measurement accuracy was examined quantitatively. 
The experimental results for gas sensitivity at different heating temperatures are shown in Fig. 1. The vertical axis of the graph shows the normalized output signal, which is defined as the difference in the resonance frequency of two resonators. When the operating temperature changes from 100 to $80^{\circ} \mathrm{C}$, the sensitivity of the sensor decreased 2.7-fold. This variation can be approximated as a straight dotted line vs temperature, and the slope of the line corresponds to a temperature coefficient of sensitivity of $0.14{ }^{\circ} \mathrm{C}^{-1}$.

Japanese Industrial Standards (JIS) stipulate the specifications for flammable gas sensors: JIS M 7653: 1996 "Portable type combustible gas detector" and JIS M 7626: 1994 "Stational type combustible gas alarm". According to this stipulation, under standard conditions (temperature $20 \pm 5{ }^{\circ} \mathrm{C}$, relative humidity $65 \pm 10 \%$, atmospheric pressure $1013 \pm 30 \mathrm{hPa}$ ), the indication error of the gas sensor must be within $\pm 25 \%$ of the actual values, and the repeatability must be within $\pm 10 \%$. Figure 2 shows a conceptual diagram of the temperature error in sensor heating. The response waveform of the temperature depends on the methods and parameters of temperature control; thus, the figure is an illustrated example. The error in sensor heating can be classified into two types: a temperature error $\left(\Delta T_{1}\right)$ between the set temperature $T_{h 0}$ and the actual temperature $T_{h}$, and a temperature fluctuation $\left(\Delta T_{2}\right)$ compared with a stable temperature. In this case, the indication error means absolute accuracy, and it is assumed that $\Delta T_{1}$ is dominant. Because repeatability is a relative parameter, $\Delta T_{2}$ can also be dominant.

By comparing the temperature coefficient of sensitivity given above with the JIS, $\Delta T_{1}$ must be within $\pm 1.8{ }^{\circ} \mathrm{C}$ to satisfy the indication error: $\pm 25 \%$. Likewise, to satisfy a repeatability of $\pm 10 \%, \Delta T_{2}$ must be within $\pm 0.7^{\circ} \mathrm{C}$.

\subsection{Energy consumption of heater}

As a design specification, the energy consumption of the sensors was estimated by preliminary experiments. Sensors with four different dimensions were manufactured, and energy consumption by the heater when the sensors were heated to $100{ }^{\circ} \mathrm{C}$ was measured. The experiment was carried out at room temperature. The aspect ratio of length $(L)$ to width $(W)$ of

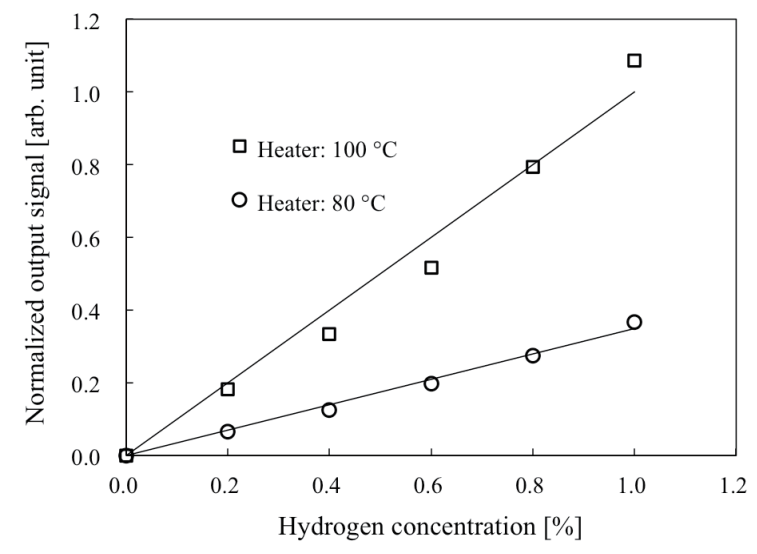

Fig. 1. Gas sensitivity at different operating temperatures.

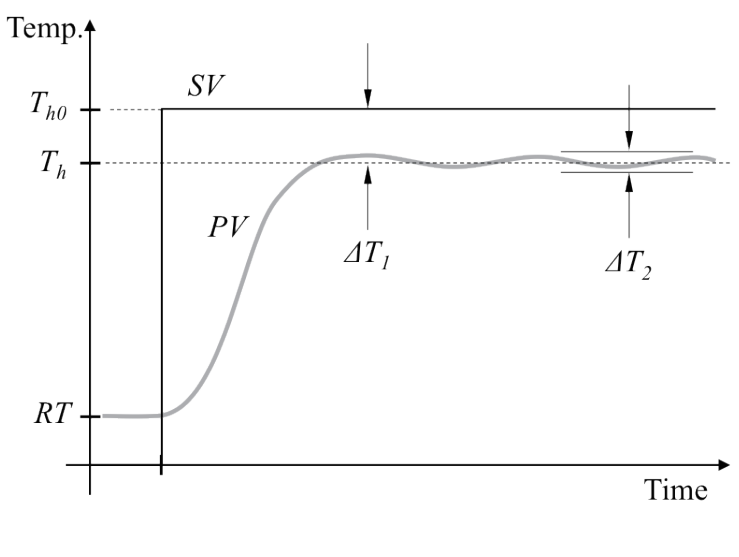

Fig. 2. Conceptual diagram of temperature error in sensor heating. 
the resonator was constant, and the rest of the design was the same. The sensor assembled on a low-temperature cofired ceramics (LTCC) package was used for the evaluation. Experimental results are shown in Fig. 3. The horizontal axis shows the surface area of the resonators. The sensor has two resonators; thus, the total surface area $S$ equals $4(L \times W)$. The thickness of the sensor is very small compared with $L$ and $W$, and it can be neglected. The experimental result indicates that the energy consumption becomes large in proportion to the surface area of the resonator. As causes of thermal losses, heat transfer and thermal radiation were considered. From a rough estimate of loss by thermal radiation, if the sensor surface is regarded as a black body, the loss by thermal radiation is three times smaller than that lost by heat transfer. Additionally, part of the sensor was made of Au thin-film electrodes that have low thermal emissivity. Therefore, the effect of thermal radiation is comparably small. In addition, the point where the extrapolated line intersects the vertical axis defines thermal loss by heat conduction. In short, heat loss is independent of the size of the sensor, and heat flux flows out into the LTCC package through the supporting part of the resonator.

As a result of the preliminary experiments, the maximum energy consumption $(S<22$ $\mathrm{mm}^{2}$ ) was identified as $200 \mathrm{~mW}$. Thus, that value was used as a design specification for the heater on the sensor. However, the power consumption of the heater changes depending on the environmental conditions, particularly ambient temperature and air flow. Thus, a margin of more than $50 \%$ should be estimated for a specification of a heater controller.

\section{Design and Fabrication}

The Au thin-film heater pattern on the sensor was designed to satisfy the requirements presented in the previous section. For the heater controller, a single voltage of $5 \mathrm{~V}$ DC was supplied. Therefore, the maximum power supplied to the heater was limited to $4 \mathrm{~V}$ and $100 \mathrm{~mA}$ (max. $400 \mathrm{~mW}$ ), which came from the specifications of commercially available op-amps. If the heater resistance was too large, the voltage drop of the heater would have exceeded $4 \mathrm{~V}$; if it was too small, the temperature of the heater would not have reached $100{ }^{\circ} \mathrm{C}$ owing to insufficient

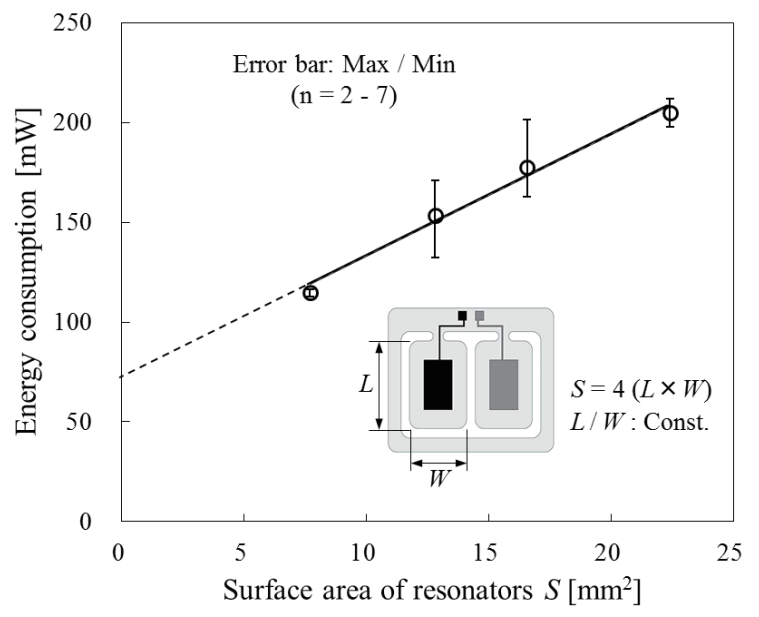

Fig. 3. Relationship between energy consumption of heater and sensor size. 
current. The range of resistance satisfying the current-voltage specification was 20 to $80 \Omega$.

Owing to the fabrication process, the film thickness of the heater must be the same as that of the excitation electrodes of the resonators. The thickness of the excitation electrodes is one of the critical parameters for the vibrational characteristic of resonators; the design of sensors, which includes quartz, excitation electrodes, and a heater, was examined using FEM simulations to optimize the energy-trapping effect of the electrodes. ${ }^{(11,12)}$ The sensor consists of two resonators, and the difference in each resonance frequency is also an important design requirement. Because the sensing resonator has the Pt catalyst on the excitation electrode, the resonance frequency of the resonator $\left(f_{s}\right)$ becomes lower than that of the reference resonator $\left(f_{r}\right)$ owing to the mass loading effect. To avoid mechanical vibration coupling between the two resonators, the frequency difference should be larger than the full width at half maximum (FWHM) of the electrical resonance curve in the frequency domain. For example, when $f_{s}, f_{r}$ $\approx 16 \mathrm{MHz}$ and $Q=1 \times 10^{4}$, FWHM $(=f / Q)$ is $1.6 \mathrm{kHz}$. Moreover, $f_{s}$ increases with hydrogen detection. A typical sensitivity of the sensor is approximately $500 \mathrm{ppm}$ for 1 vol.\% hydrogen. Thus, a frequency increase of $32 \mathrm{kHz}$ is estimated at a hydrogen concentration of $4 \mathrm{vol} . \%$. For this reason, the thickness of the Pt catalyst is regulated so that the differences in the resonance frequency exceed $50 \mathrm{kHz}$.

In addition, it is well known that the resistivity of thin-film metals is higher than that of bulk metals. The dominant factor in resistivity changes on thin films is grain size. In the case of sputtered films, sputtering conditions such as RF power, pressure in the vacuum chamber, substrate temperature, and film thickness, are all parameters that determine resistivity. In reference to previous studies, the resistivity of Au thin films of 5-10 $\mu \Omega \cdot \mathrm{cm}$, which is 2- to 4-fold higher than that of the bulk, has been reported. ${ }^{(13-15)}$ In this study, a resistivity 2.43 -fold higher, which was an experimentally determined coefficient, is used for designing the heater pattern. The resistances of the heater patterns were $44.2 \Omega$ at $25^{\circ} \mathrm{C}$ and $50.0 \Omega$ at $100{ }^{\circ} \mathrm{C}$.

The fabrication process for the sensor with a $\mathrm{Au}$ thin-film heater is shown in Fig 4. In this process, the heater was patterned at the same time as the excitation electrodes of the resonator. The shape of the heater is linear and it is arranged around the excitation electrode.
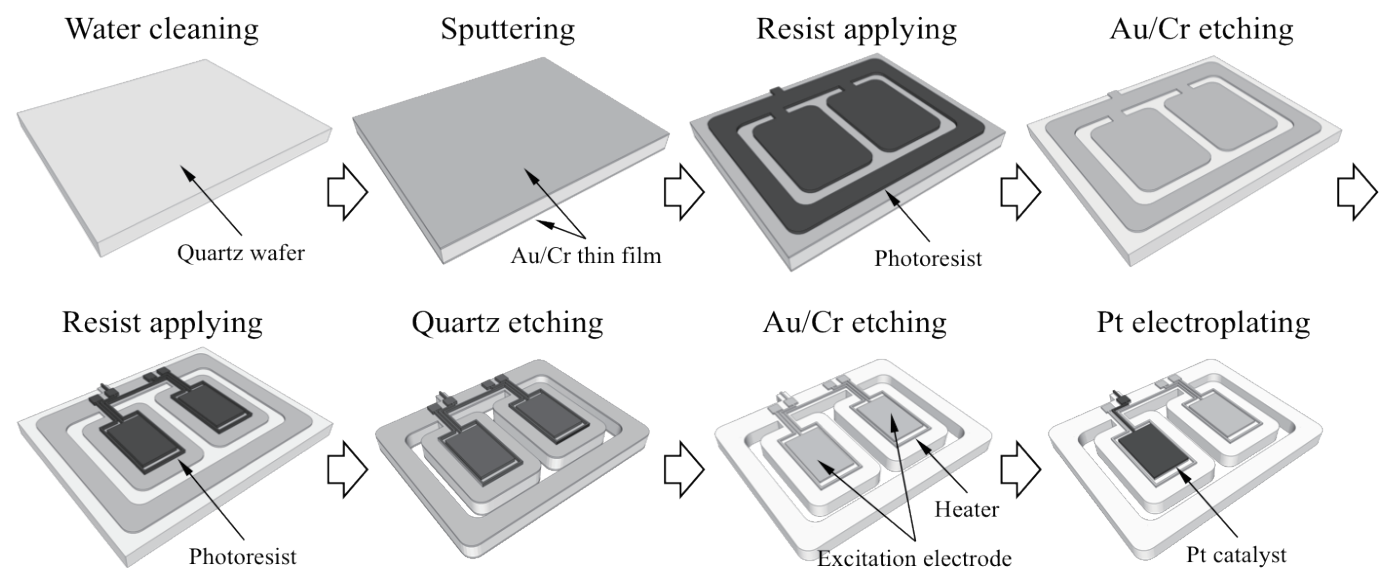

Fig. 4. Fabrication process for the sensor with the Au thin-film heater. 
The excitation electrodes were patterned on both sides of the quartz surfaces, but the heater was patterned on the upper surface only. This process includes two photolithography steps, the first one for patterning the quartz outline, and the second one for patterning the electrodes and heater. In order to minimize heat conduction between the two resonators, the resonator part was completely separated except the beam portion at the quartz etching process.

A comparison of this fabrication process to that of the previous sensor with a Pt thin-film heater is shown in Figs. 5(a) and 5(b). The lithography is done by a wet process. Because the Pt thin film cannot be etched by a wet process, a lift-off process had to be used. Compared with the processes for the new sensor, the previous process required three additional processes related to lift-off. ${ }^{(7)}$ Therefore, shortening of the fabrication process is a strong advantage. Moreover, in mass production, achieving a high yield rate is difficult with a lift-off process, and sputtering of $\mathrm{Pt}$ also incurs a high cost. Consequently, changing the heater material from $\mathrm{Pt}$ to $\mathrm{Au}$ has the potential to provide significant cost savings for the sensor.

Figure 6 shows a photograph of the manufactured sensor. The LTCC package has cavities on both surfaces. In the upper cavity, a sensor is mounted, and a metal mesh is attached as a particle filter. Additionally, some electrical components of the oscillation circuit and a flexible flat cable are mounted on the lower cavity.

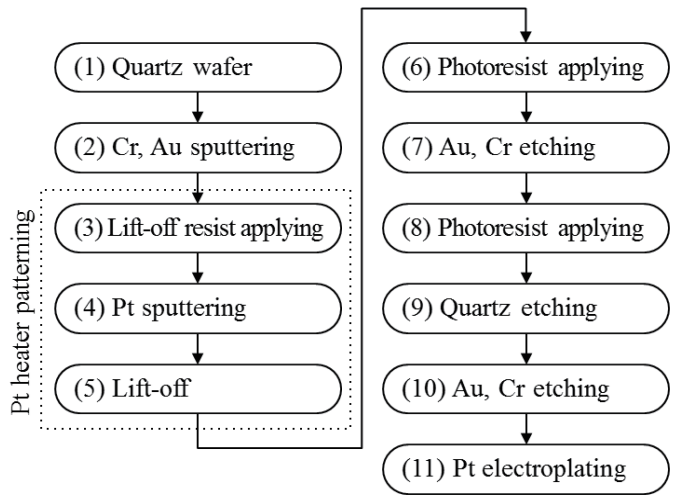

(a)

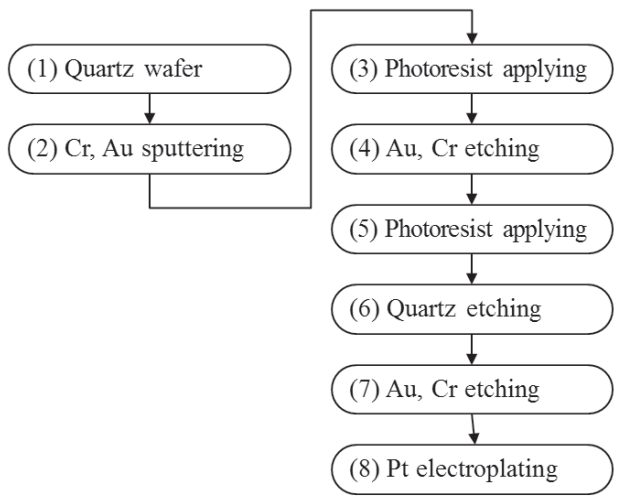

(b)

Fig. 5. Comparison of fabrication processes for (a) previous sensor with Pt thin-film heater and (b) newly designed sensor with Au thin-film heater.

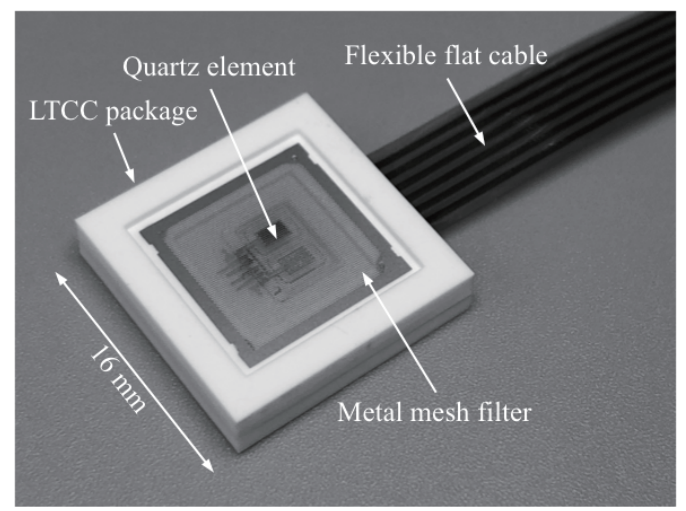

Fig. 6. Photograph of the newly designed hydrogen sensor. 


\section{Characterization of Au Thin-film Heater}

The temperature characteristics of the heater of the manufactured sensor were evaluated. Using a thermostatic chamber, the temperature was changed from room temperature to $120{ }^{\circ} \mathrm{C}$ in $20{ }^{\circ} \mathrm{C}$ increments, and, for each temperature, a waiting time of $20 \mathrm{~min}$ was included. The temperature profile measured by a thermocouple placed adjacent to the sensor is shown in Fig. 7(a). The heat cycling was repeated 5 times. A high-precision digital multimeter (Agilent 3458A) was used, and a four-terminal resistance measurement method was adapted to eliminate errors due to read wires. The experimental results are shown in Fig. 7(b). The results of the first cycling and the others do not match clearly. Figure 7(c) shows the properties of the heater during heat cycling. The vertical axis on the left shows the resistance of the heater at $100{ }^{\circ} \mathrm{C}$, and the vertical axis on the right shows the temperature coefficient of resistance (TCR) of the heater. Because both graphs show an exponential trend, it is assumed that the structure of the thin film was changed by heating. In general, the grain size of a thin film grows because of annealing, and its properties approach those of the bulk. However, the experimental data on resistance and TCR trended in the opposite direction. Because the heater consisted of laminated thin films of $\mathrm{Au}$ and $\mathrm{Cr}$, the diffusion of $\mathrm{Cr}$ grains into the $\mathrm{Au}$ layer may have been considered.

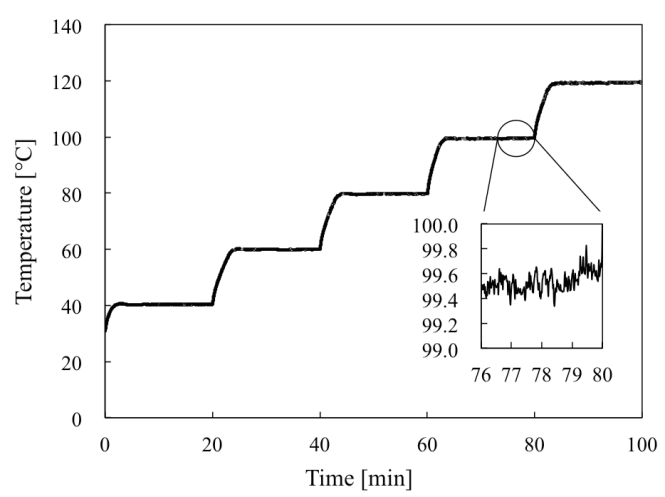

(a)

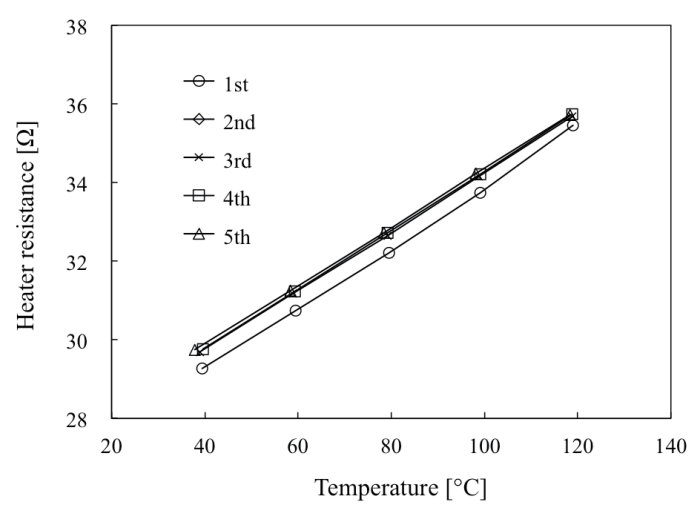

(b)

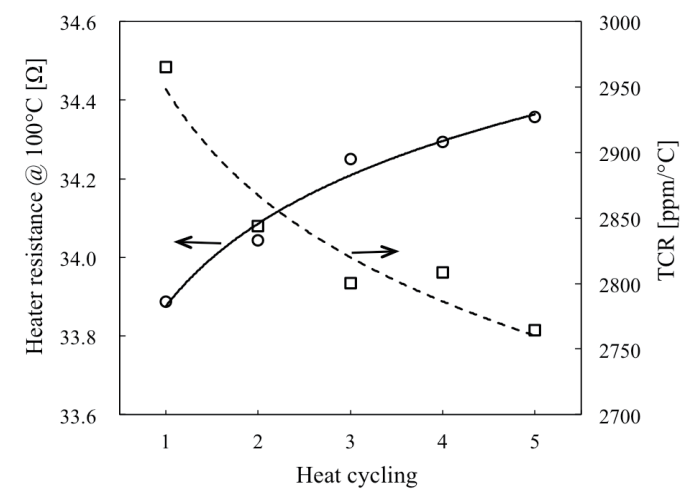

(c)

Fig. 7. (a) Condition of temperature test. (b) Temperature characteristics of heater with heat cycling. Resistance and TCR changes with heat cycling. 
In a previous report, we demonstrated the result of the effective annealing of a Au thinfilm heater at a temperature of $150{ }^{\circ} \mathrm{C} .{ }^{(9)}$ However, because of the storage temperature of ICs mounted on the LTCC package, the highest annealing temperature of the sensor was limited to $125{ }^{\circ} \mathrm{C}$. Therefore, continuous annealing at $120{ }^{\circ} \mathrm{C}$ was tested. A sensor without a history of heating since its soldering (peak reflow temperature: $220-230{ }^{\circ} \mathrm{C}, 10 \mathrm{~s}$ ) in the packaging process was used. The experimental result is shown in Fig. 8. The resistance of the heater suddenly changed in the first $5 \mathrm{~h}$, and after that, it turned to the saturation trend. However, even after $5 \mathrm{~h}$, the resistance change continued slightly. Thus, the stabilizing time for the heater was determined as $24 \mathrm{~h}$, where the resistance change rate is less than $5 \%$ to the target temperature accuracy.

The stabilizing time on the annealing process changes depending on the thicknesses of the $\mathrm{Au}$ and $\mathrm{Cr}$ films. In this study, the thickness of $\mathrm{Au}$ is $300 \mathrm{~nm}$ and that of $\mathrm{Cr}$ is $20 \mathrm{~nm}$. If thicknesses of the thin films are small, the annealing time can be shortened. However, to obtain good vibrational characteristics of the resonators, the film thicknesses have been predetermined.

\section{Development of Heater Controller}

In order to realize the inexpensive sensors, it is necessary to minimize the number of parts of the drive circuit and to make the area of the printed circuit board small. Therefore, we designed a very simple control circuit to achieve constant heating by the heater shown in Fig. 9. The controller consists of a bridge circuit, a detection amplifier, and an integrator. For the circuit, a single-supply, rail-to-rail input/output amplifier was used. The balance condition of the bridge circuit is represented by Eqs. (1) and (2).

$$
\begin{gathered}
R_{h}(T)=R_{h 0}\left[1+\alpha\left(T-T_{0}\right)\right], \\
R_{h}(T)=\frac{R_{1}}{R_{2}}\left(R_{3}+R_{4}\right),
\end{gathered}
$$

where $R_{h}$ is the resistance of the heater at any temperature $T, R_{h 0}$ is the resistance of the heater at the reference temperature $T_{0}, \alpha$ is TCR of the heater, $R_{1}, R_{2}$, and $R_{3}$ are the resistances of

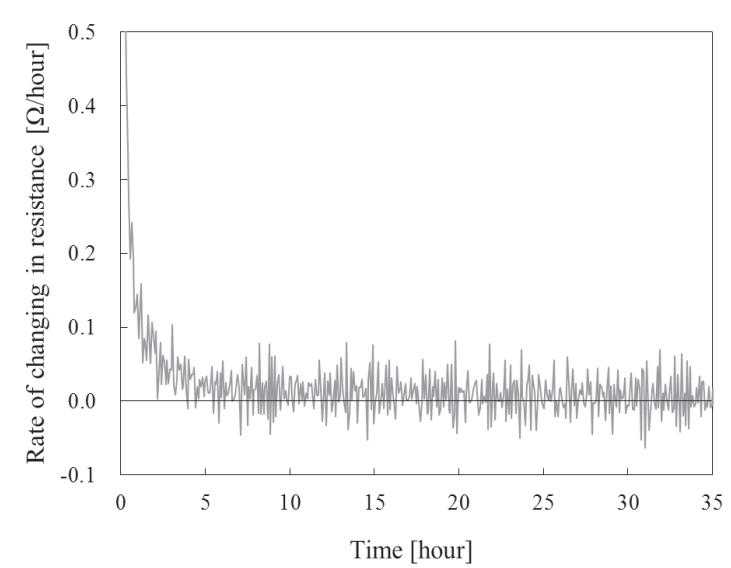

Fig. 8. Effect of annealing on Au thin-film heater at $120^{\circ} \mathrm{C}$.

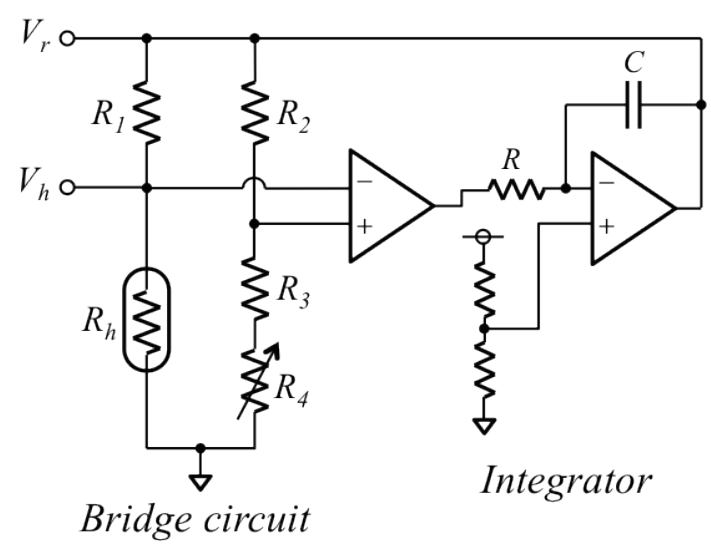

Fig. 9. Circuit diagram of heater controller. 
fixed resistors, and $R_{4}$ is the resistance of a variable resistor. The resistance of the heater can be detected by the bridge circuit and amplifier. At the beginning of heating, $R_{h}$ is smaller than the balance condition, and the output of the detecting amplifier is positive. Then, a positive voltage $V_{r}$ is applied at the bridge apex. When the heater reaches the desired temperature, the balance conditions of the bridge are satisfied. For example, by decreasing the heat discharge by increasing the environmental temperature, when the temperature of the heater becomes higher than a desired value, the output of the amplifier is inverted, and then $V_{r}$ becomes smaller.

On the other hand, the integrator has two roles. The first role is as a slow start function to reduce the response speed during heating. In the absence of the integrator in the feedback, maximum current is supplied to the heater when the power is turned on. Then, a hot spot appears at a place where the electric field concentrates locally such as the vertex of the heater pattern, and significant high thermal stress is generated at that area in the quartz substrate. In previous experiments, we observed destruction in the quartz substrate due to thermal stress when a controller had no current-limiting function. The second role is to avoid abnormal oscillations in the negative feedback loop. The time constant was determined on the basis of experimental evaluation.

Figure 10 shows the response wave of the heater resistance during heating. The target resistance of the heater was adjusted to $51.03 \Omega$ using the variable resistor $R_{4}$. The resistance of the heater is obtained from the partial voltages $V_{r}$ and $V_{h}$. In the graph, heating was started at time $t=10 \mathrm{~s}$. As a result, the resistance of the heater becomes balanced after $250 \mathrm{~s}$. The electrical characteristics of the heater at the steady state are shown in Table 1. The measured resistance was $51.04 \Omega$, and the calculated temperature of the heater was $100.16{ }^{\circ} \mathrm{C}$. All the electrical parameters such as voltage, current, and energy consumption were satisfied by the design specification previously explained. The temperature response of the heater is shown in Fig. 11. On this graph, two data sets are plotted for comparison. One is the temperature calculated from heater resistance, the other is the temperature calculated from the resonance frequency of the reference resonator. The measured values obtained from the two different methods match perfectly in the steady state; its data support the accuracy of the measurements. In contrast, a slight disagreement is seen in the transition period. The heater-calculated value

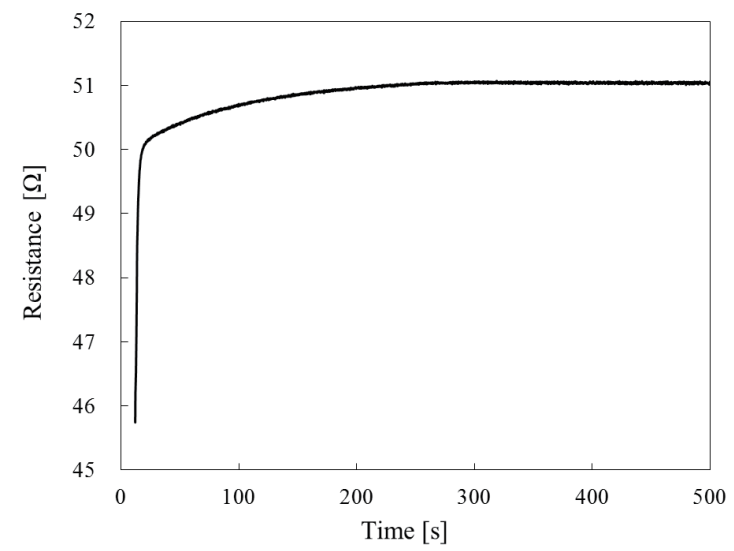

Fig. 10. Response curve of heater resistance using the heater controller.
Table 1

Electrical characteristics of the heater at steady state $(t=400-500 \mathrm{~s})$.

\begin{tabular}{lccc}
\hline & Average & SD $(\sigma)$ & Max. - Min. \\
\hline Voltage $V_{h}(\mathrm{~V})$ & 3.19 & - & - \\
Current $(\mathrm{mA})$ & 62.54 & - & - \\
Heater resistance $(\Omega)$ & 51.04 & 0.01 & 0.06 \\
Temperature $\left({ }^{\circ} \mathrm{C}\right)$ & 100.16 & 0.12 & 0.69 \\
Energy consumption $(\mathrm{mW})$ & 199.6 & - & - \\
\hline
\end{tabular}


is the temperature of the quartz surface, and the resonance-frequency-calculated temperature is the average temperature of the quartz resonator. Thus, a temporary temperature distribution may have occurred on the surface and in the core of the quartz.

Table 2 shows the resonance frequencies of the resonators at steady state. The frequency deviation was calculated assuming that the resonance frequency at room temperature was zero. Here, the resonance frequencies at $100{ }^{\circ} \mathrm{C}$ are $f_{s}=15.782 \mathrm{MHz}$ for the sensing resonator and $f_{r}$ $=15.845 \mathrm{MHz}$ for the reference resonator. The difference in frequency deviations is caused by a difference in TCF. The TCF of the quartz wafer was the same for both resonators. However, owing to the influence of attached electrodes, it was changed slightly. ${ }^{(16)}$

Figure 12 shows the experimental results of sensor output in a hydrogen concentration range of $0-2$ vol.\%. Pure hydrogen gas and standard dry air were used as test gases, and mass flow controllers were used to control the concentration of the test gas. The difference in the frequency deviations of each resonator $\left(\Delta f_{s}-\Delta f_{r}\right)$ was defined as sensor output signal, and the reference point of zero for the output was reset to the resonance frequency at $100{ }^{\circ} \mathrm{C}$. In the graph, the results obtained from two different heating methods are compared. One is the sensor output using the heater controller; the other is the sensor output using a current source. Here, $n$ means the number of samples, and the data from the output using the heater controller was the average value of the different sensors. The result using the current source is curved. We suggest that the temperature of the heater was increased by the energy generated by the catalytic reaction, and the temperature of the sensor was increased by the increasing heater

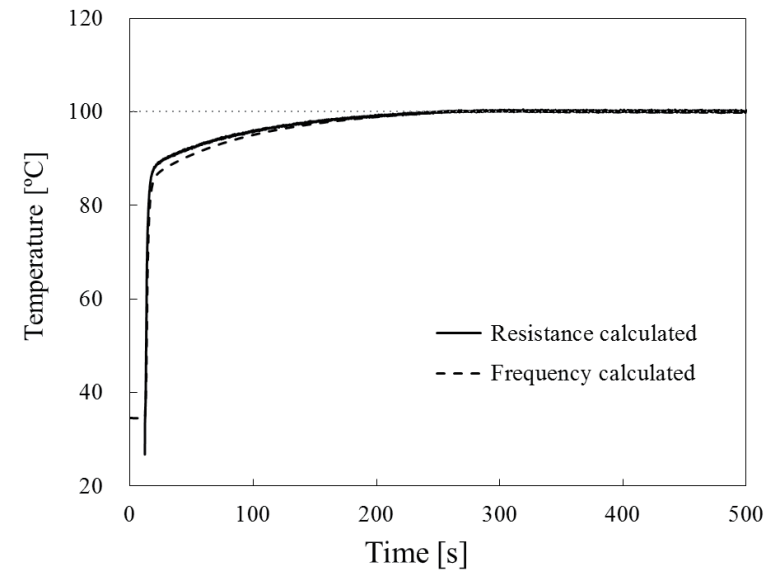

Fig. 11. Comparison of temperatures rising in the sensor: temperature calculated from heater resistance and temperature calculated from resonance frequency.

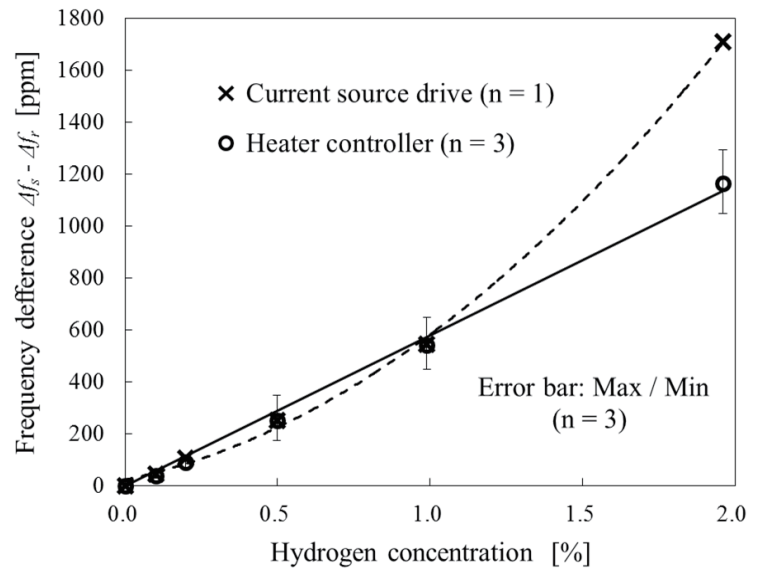

Fig. 12. Comparison of calibration curves of the sensor by different heating methods.

Table 2

Resonance frequency of resonators at steady state $(t=400-500 \mathrm{~s})$. $Q_{s}$, average $Q$ factor of sensor resonator within the same lot; and $Q_{r}$, average $Q$ factor of reference resonator within the same lot.

\begin{tabular}{lccc}
\hline & Average & SD $(\sigma)$ & Max. - Min. \\
\hline Sensor resonator $\left(Q_{s}\right)$ & $8.4 \mathrm{k}$ & - & - \\
Reference resonator $\left(Q_{r}\right)$ & $12.1 \mathrm{k}$ & - & - \\
Sensor resonator $\left(\Delta f_{s}\right)$ & $1330 \mathrm{ppm}$ & $0.26 \mathrm{ppm}$ & $0.86 \mathrm{ppm}$ \\
Reference resonator $\left(\Delta f_{r}\right)$ & $1453 \mathrm{ppm}$ & $0.55 \mathrm{ppm}$ & $2.22 \mathrm{ppm}$ \\
Difference $\left(\Delta f_{s}-\Delta f_{r}\right)$ & - & $0.50 \mathrm{ppm}$ & $1.80 \mathrm{ppm}$ \\
\hline
\end{tabular}


resistance. In short, the heater becomes an unstable positive feedback controller. On the other hand, the result using the heater controller shows a linear relation, and a strong correlation between the experimental data and the calibration curve approximated by a regression line is obtained. Therefore, the result demonstrates an improvement in the accuracy and stability of the sensitivity by using the new controller.

Of course, it is possible to precisely adjust the heater temperature to the target by the fine adjustment of the current source output. However, this balance point of temperature will be changed due to the change in the outside gas temperature and its flow velocity surrounding the sensor. Although this problem can be solved by some methods such as controlling the output of the current source by detecting the voltage of the heater, it becomes probably more complicated than our designed circuit. Therefore, it does not lead to the realization of an inexpensive and compact gas sensor.

\section{Discussion}

This development was carried out to realize stable sensor heating. A comparison between the objectives set according to JIS and the results is shown in Table 3. The experimental results achieved 10-fold smaller $\Delta T_{1}$ and more than 2-fold smaller $\Delta T_{2}$. The experimental data were obtained under room temperature conditions. Temperature test conditions written in the JIS are regulated as an environmental temperature of $20 \pm 20{ }^{\circ} \mathrm{C}$. Therefore, the effect on the sensitivity under a wide-environmental-temperature environment should be discussed. The temperature of the heater may be adjusted by changing the values of the resistors used in the bridge circuit. Each resistor has its respective TCR, and the resistances of all the resistors are changed by changing the temperature. That effect can be minimized by using high-precision resistors. In contrast to fixed resistors, the TCR of commercially available variable resistors is 100 to $500 \mathrm{ppm} /{ }^{\circ} \mathrm{C}$, which is up to several tens of times larger than that of high-precision fixed resistors. As an analytical result, over a temperature range of $\pm 60^{\circ} \mathrm{C}, \Delta T_{1}$ in a worst-case scenario is only $1.2{ }^{\circ} \mathrm{C}$. Even if the environmental temperature changed over a range of $20 \pm 20^{\circ} \mathrm{C}$, $\Delta T_{1}$ can theoretically be kept in the desired range. However, this should be verified in a future work.

In addition, we discussed the stability of the output signal to estimate the resolution in hydrogen concentration detection. The average sensitivity given by the calibration curve of the sensor was $578.6 \mathrm{ppm} / \mathrm{vol} . \%$. On the other hand, the standard deviation $\sigma$ of the different values of the resonance frequencies was $0.50 \mathrm{ppm}$, and this can be regarded as the noise level on a baseline of frequency. From these values, the limit of detection (LOD, $3 \sigma)$ is 0.0026 vol.\% (= 26 $\mathrm{ppm}$ ), and the limit of quantitation (LOQ, $10 \sigma$ ) widely used in measurement field is 0.0086 vol.\% (= $86 \mathrm{ppm}$ ). Therefore, we conclude that the detection of a hydrogen concentration of $0.01 \mathrm{vol} . \%$ is sufficiently possible by using techniques such as the moving average method.

Table 3

Comparison between objectives and result of heater control.

\begin{tabular}{lcc}
\hline & Objective & Result \\
\hline Temperature error $\Delta T_{1}\left({ }^{\circ} \mathrm{C}\right)$ & $\leq \pm 1.8$ & \pm 0.16 \\
Temperature fluctuation $\Delta T_{2}\left({ }^{\circ} \mathrm{C}\right)$ & $\leq \pm 0.7$ & $+0.47 /-0.22$ \\
\hline
\end{tabular}


In this study, the waiting time for temperature stabilization after heating was excluded from the subject of discussion, because the requirement depends on sensor applications. As typical requirements for start-up time, it should be within a few seconds for fuel cell vehicles, and within $30 \mathrm{~s}$ for portable gas detectors. For a stationary gas detection system, the start-up time is not important, because sensors are used during 24-h drives.

\section{Conclusions}

In this paper, we focus on heating technologies for catalytic combustion gas sensors using quartz resonators. To present the requirements for heating accuracy of the sensor quantitatively, the effect of temperature error on the sensitivity of the sensor was analyzed. A fabrication process for the newly designed sensor with a Au thin-film heater was introduced, and advantages in the process were presented. The characteristics of the Au thin-film heater of the sensor were evaluated. Using the experimental results, the accuracy of measuring the heating temperature was discussed. Temperature error with respect to a target value and fluctuations during a period of steady state fell within the targeted range, which was determined by referring to the JIS for catalytic combustion gas sensors. Additionally, as a result of sensitivity measurements using the designed heater controller, a constant sensitivity in the range of 0-2 vol.\% was obtained. Because LOQ on hydrogen detection in air was $86 \mathrm{ppm}$, we conclude that the hydrogen gas sensor can detect a concentration of 0.01 vol.\% using the proposed stable heating technologies.

\section{Acknowledgments}

This paper is based on results obtained from a project commissioned by the New Energy and Industrial Technology Development Organization (NEDO).

\section{References}

1 L. Boon-Brett, J. Bousek, G. Black, P. Moretto, P. Castello, T. Hübert, and U. Banach: Int. J. Hydrogen Energy 35 (2010) 373.

2 W. J. Buttner, M. B. Post, R. Burgess, and C. Rivkin: Int. J. Hydrogen Energy 36 (2011) 2462.

3 I. Takahashi: J. Surf. Finish. Soc. Jpn. 57 (2006) 267 (in Japanese).

4 K. Tsubota, T. Kiwa, H. Yamada, T. Yamaguchi, Y. Kondo, H. Imajo, T. Hondo, T. Maehara, T. Yamamoto, and K. Tsukada: IEEJ Trans. Sens. Micromach. 129 (2009) 278.

5 W. Shin, M. Matsumiya, N. Izu, and N. Murayama: Sens. Actuators, B 93 (2003) 304.

6 H. Oigawa, D. Yamazaki, and T. Ueda: IEEJ Trans. Sens. Micromach. 131 (2011) 117 (in Japanese).

7 H. Oigawa, Y. Kirino, D. Yamazaki, and T. Ueda: Proc. 5th Int. Conf. Sensing Technology (2011) 587.

8 H. Oigawa, K. Harima, F. Kohsaka, T. Tsuno, and T. Ueda: Proc. IEEE Sensors 2016 (IEEE, 2016).

9 H. Oigawa, M. Shimojima, T. Tsuno, F. Kohsaka, and T. Ueda: Proc. 2017 Eleventh Int. Conf. Sensing Technology (2017) 209.

10 D. Yamazaki and T. Ueda: IEEJ Trans. Sens. Micromach 130 (2010) 65.

11 W. Shockley, D. R. Curran, and D. J. Koneval: J. Acoust. Soc. Am. 41 (1967) 981.

12 J. Ji, H. Oigawa, H. H. Chen, M. Zhao, and T. Ueda: IEEJ Trans. Sens. Micromach. 132 (2012) 275.

13 D. Hecht and D. Stark: Thin Solid Films 238 (1994) 258.

14 M. Adamov, B. Perović, and T. Nenadović: Thin Solid Films 24 (1974) 89.

15 R. B. Belser and W. H, Hicklin: J. Appl. Phys. 30 (1959) 313.

16 O. Ishii, H. Iwata, M. Sugano, and T. Shibata: IEICE Trans. Commun. 98 (1998) 19. 